\title{
Towards metering of Tap water by Lorentz force velocimetry: Realization and improvement of non-contact flow measurement method
}

\author{
Suren Vasilyan, Thomas Fröhlich \\ Institute of Process Measurement and Sensor Technology, \\ Technische Universität IImenau, 98684 \\ IImenau, Germany \\ Suren.vasilyan@tu-ilmenau.de
}

\begin{abstract}
The present work describes recent improvement on non-contact/non-intrusive flow measurements method by means of Lorentz Force Velocimetry. Particularly, flow metering in channel of purely conducting fluid in range of $10^{-2}$ to $10^{1} \mathrm{~S} / \mathrm{m}$ conductivities and 0.2 to $4 \mathrm{~m} / \mathrm{s}$ velocities are discussed. Method is realized based on high resolution measurements of single axis horizontal directed forces. These are reaction forces that are acting to the magnet system as a result of induced Lorentz force due to transverse interaction of magnetic field of magnets and velocity field of the flow. Test measurements of forces, which are linearly proportional to conductivity and velocity, are conducted by differential force measurement system developed based on State of the Art ultra-precision balances. We present measurements in order of $10^{-8}$ to $10^{-6} \mathrm{~N}$ as a function of electrolyte flow rate and corresponding sensitivity considerations. Typical measurement range of $\approx 4 \times 10^{-6} \mathrm{~N}$ forces corresponding to the flow of saline water with conductivity of $1 \mathrm{~S} / \mathrm{m}$ and velocity of $1 \mathrm{~m} / \mathrm{s}$ in a rectangular duct with a $50 \times 50 \mathrm{~mm}$ cross-section. The precision and stability of measurement signal is examined under environmental influences such as thermal radiation and ground vibration and their reduction mechanisms are discussed respectively.
\end{abstract}

Key words: flow measurement, force measurement, Lorentz Force Velocimetry, electromagnetic force compensation, tilt measurements

\section{Introduction}

Recent advance in flow metering of electrolytes through contactless method of Lorentz Force Velocimetry (LFV) [1] demands application specific developments of high precision Force Measurement Systems (FMS). Theoretical ground for LFV was derived by the classical discipline, in Magnetohydrodynamics (MHD) originally proposed by J.A.Shercliff [2], that is when a flow of an electrically conducting fluid interacts (relative motion) with magnetic field, eddy currents are produced (also known as secondary current) within the bulk of the fluid. These currents in turn interact with the magnetic field giving rise to the Lorentz force that breaks the fluid. In accord to Newton's 3rd law, an equal but opposite force act on the magnet. Measurements of these forces is the key ingredient of LFV which can be done up to certain extend with existing State of the Art or developed application specific force and torque measuring systems [3-7].
The Lorentz force (LF) over volume of the test section of channel Eq. 1 is linear dependent and unidirectional to mainly horizontal directed vector of mean flow velocity, meanwhile linear dependence to conductivity of electrolyte in our study is set to be constant for each set of experimental measurements.

$$
\begin{aligned}
& \vec{F}_{L}=\int_{V} d V \cdot \vec{j} \times \vec{B} \\
& F_{L} \sim \sigma \cdot v_{0} \cdot B^{2}
\end{aligned}
$$

Here, $V$ is the volume of interaction of magnetic field $\mathrm{B}$ and mean velocity field $v_{0}$ of fluid in the bulk of the electrolyte (conductivity $\sigma$ ) where eddy currents $j$ are generating.

From (1b) can be drawn a simplifying expression for this measurements in terms of calibration factor 
$c \cdot B^{2}=\frac{F_{L}}{\sigma \cdot v_{0}}$

$c$ - is a constant factor of each particular experimental setup that depends on several factors. Mainly dependent from active interaction region of $\vec{B}$ and $\vec{v}_{0}$, for the constant a) physical shape and properties of construction, b) characteristic dimensions of the channel, and finally c) geometry of magnet system and its magnetic field distribution are of primary importance. In the end of this paper we provide calibration factor of our particular experimental facility, which is the value somewhat defining sensitivity and accuracy of the measurement setup.

Initially, in this work we reexamine previous studies and developments based on $[4,7]$ in terms of technological implementation of measurement scheme. This led us to consideration of environmental influences on measurement precision and signal stability. Further we present a differential force measurement method, in order to suppress those influences, which provide nearly 2 order of magnitude higher resolution measurements. Subject to chosen construction of supporting housing, at least for short term period, measurement resolution within limits of State of the Art devices is achieved. The State of the Art devices are a commercially available ultraprecision weighing balance base on Electromagnetic force compensation (EMC) principle with resolution of $1 \mathrm{ug}$ provided by Sartorius [8] (1ug weight corresponds to $\approx 10 \mathrm{nN}$ force).

Conceptually, the character of measurements are dictated by the use of system of high power permanent magnets, weighing $\approx 1 \mathrm{~kg}$ and arranged from FMS, so that they produce onto FMS $\approx 10 \mathrm{~N}$ unavoidable vertical load and are located in immediate vicinity of electrolyte flow channel encompassing it from both sides with minimal air gap (see Fig. 1). In contrast to the measurand - Lorentz Force - , which is in horizontal direction and typically ranges as $10^{-8}$. $10^{-5} \mathrm{~N}$, the dead load of magnets contributes dramatically on character of force signal. As main objective of the research is measurement realization of Lorentz forces under these conditions.

In following section mechanical system based on single EMF will be analyzed in terms of temperature influences and differential measurement setup will be introduced. Further electrolyte flow measurements for various values of conductivities will be shown and finally conclusions concerning flowmeter's sensitivity provided.

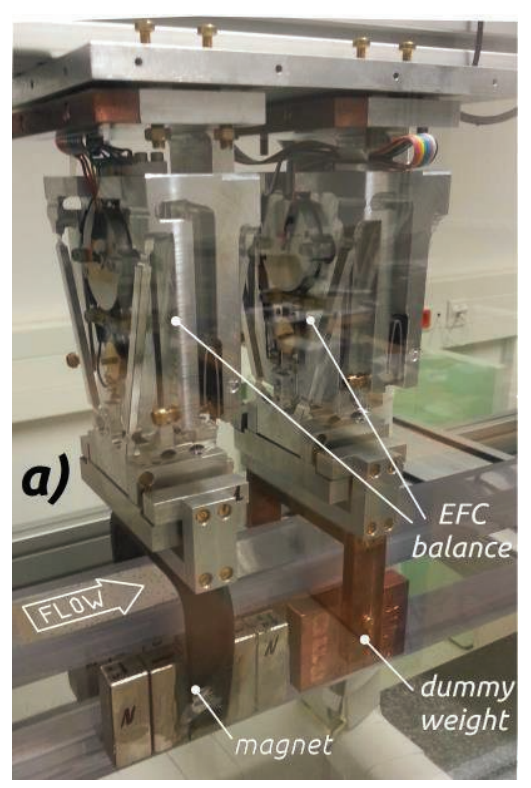

Fig. 1. Image of differential force measurement system. Encompassing walls of the channel with $1 \mathrm{~mm}$ air gap.

\section{Mechanical system and temperature influences}

Measurements presented in [4] have been conducted with the single EMC system [7] of around $1 \mathrm{uN}$ force measurement resolution. In this system with typical measurements of $\approx 4 \times 10 \mathrm{uN} \pm 1 \times 10 \mathrm{uN}$ (standard deviation) forces one can map the flow velocity of saline water with $1 \mathrm{~m} / \mathrm{s}$ while conductivity is $1 \mathrm{~S} / \mathrm{m}$ and rectangular duct has $50 \times 50 \mathrm{~mm}$ cross-section area. Based on this results further objectives were made; for the first increasing resolution of next class of force measurement devices. Also, for mid- and long term period system was reluctant to carry stable measurements (random drift of force signal was observed) therefore as a second objective increasing stability of measurement signal was identified.

Noise, however, interfering in measurement system as a mechanical disturbance, is main limitation factor to this extent and is in particular focus of our study. Careful monitoring of the force signal over successive attempts by short-, mid- and long-term period shows that besides instantaneously occurring purely mechanical (ground/seismic) vibration noises there is drift which has relatively long term character.

In turn, caused by temperature fluctuations of ambient environment, supporting construction of the force measurement setup experiences thermal expansion that leads the setup to tilt in relation to its zero equilibrium point. We employ 
test scheme (see Fig.2) to demonstrate experimentally the influence of tilt on total measurement force based on the single EMC setup presented in [7], and reference tilt meter [9] aligned to common $X$ and $Y$ axes.

Influence of thermal expansion effect on measurements has typical drift as shown in Fig.2b, this is achieved applying artificial heat source on support of measurement setup. Parallel measurements by reference tilt meter [9] shows predicted range of error given in [7]. In general for given temperature distribution and simplified construction of setup this complex behaving error can be theoretically calculated.
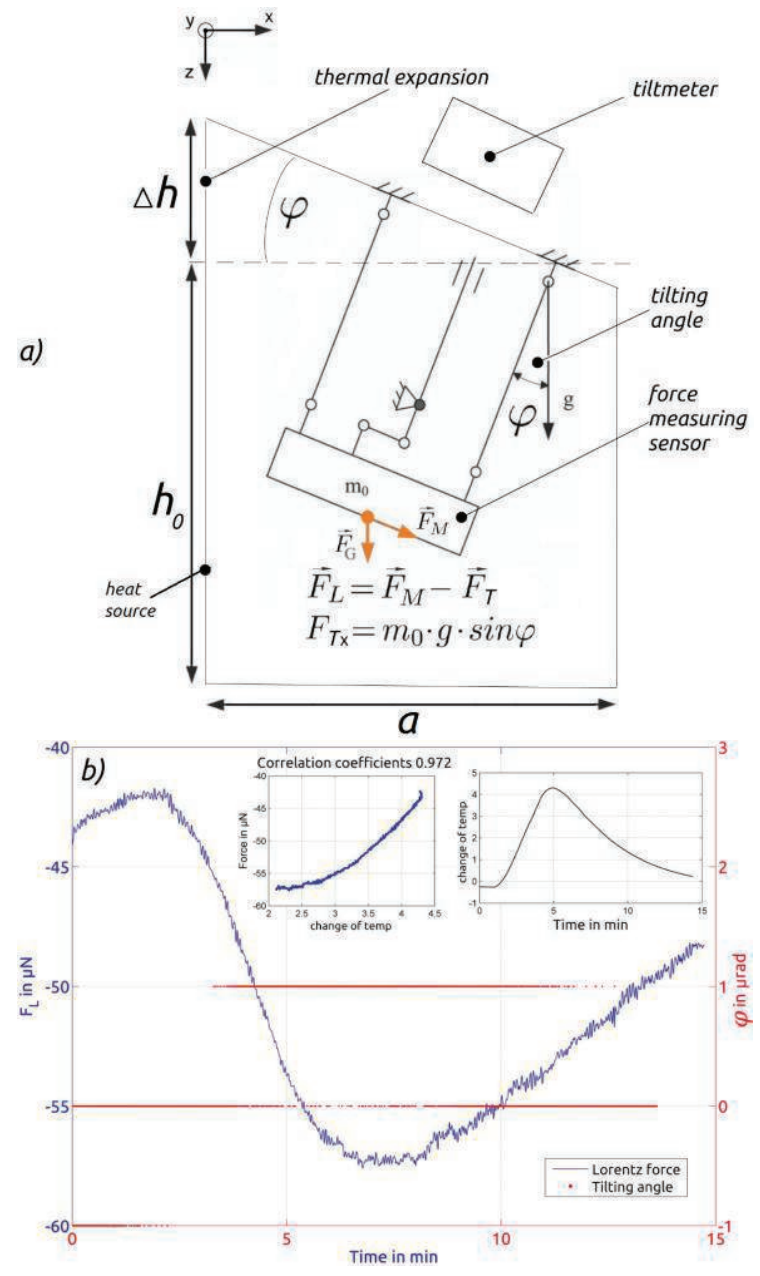

Fig. 2. a) schematic diagram of tilting error: $F_{G}$ gravity force, $m_{0}$-dead load the mass of magnets, $g$ gravitational acceleration. b) Measurement of force signal in $\mu \mathrm{N}$ (solid line) over 15 minute. Inclination/tilt of force setup (horizontal dotted lines) in $\mu \mathrm{rad}$. Inset in right change of temperature and left correlation of force signal from initial 5 minutes temperature change.

When we consider expansion only in vertical direction (see Fig.2a) and respectively influence only in $X Z$ plain then one can compute tilting angle as $\varphi=\tan \left[\frac{\Delta h}{a} \cdot \alpha_{A l} \cdot \Delta T\right]$

Where $a$ is characteristic base length, $\alpha_{A l}$ coefficient of thermal expansion of aluminum, $\Delta T$ - temperature change.

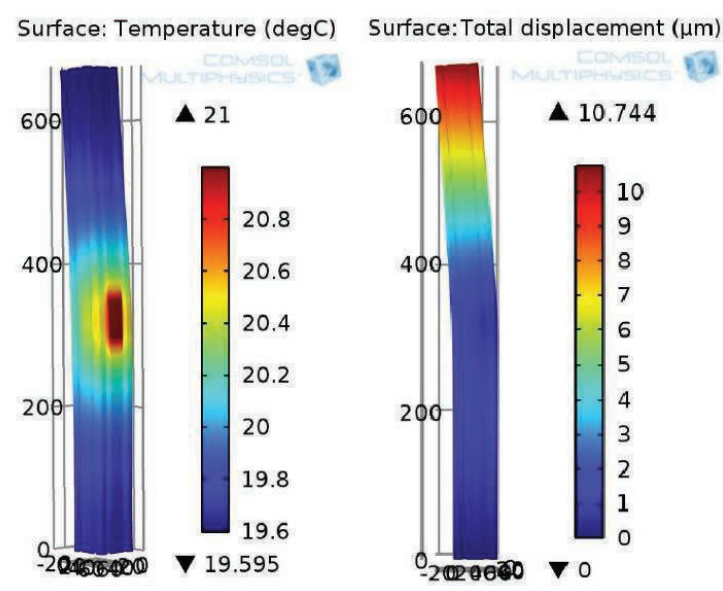

Fig. 3. Simulations in Comsol [10] shows rough estimations about the nature of thermal drift. Temperature change in $\approx 2 K$ causes around $10 u m$ total displacement $(\Delta h$ in $X Y Z)$ whereas $h \approx 700 \mathrm{~mm}$.

Since the temperature is distributed nonuniformly in the laboratory and due to technological peculiarities to dump any thermal source, computing or predicting thermal expansion effect on relatively big size of construction becomes complex problem. Instead, from practical point of view, to suppress tilt error and eliminate other vibrational noises we introduce second identical EMC balance with dummy weight to mimic all secondary influences but - electromagnetic interaction of flow and magnets (see Fig.1). Next, construction and measurement results of differential measurement method will be presented.

\section{Differential force measurement system}

The core of setup is two identic EMC balances. Technically the balance is capable to measure single axis force in $\pm 55 \mathrm{mN}$ range with $10 \mathrm{nN}$ resolution (corresponds to values provided by manufacturer that is $\pm 5.5 \mathrm{~g}$ range with $1 \mathrm{ug}$ readability). Both EMC balances are aligned to $X Y$ plane in a way to measure forces in direction of $X$ horizontal axis. Since development of the noise in measurement process has random multi axis behavior, we aimed to construct a setup with minimal technical complexity. This is done to keep nonlinear and multi axis influences on to the setup similar in order to analyze them better and to have understanding about their origin. Schematic diagram (see Fig. 4) presents 
simplified construction of image in Fig. 1 and a bit exaggerated measurement principle of Lorentz force $F_{L}$ and respective error influences (the ground vibration and thermal drift) $F_{e r r}$ meanwhile existing in both EMC balances.

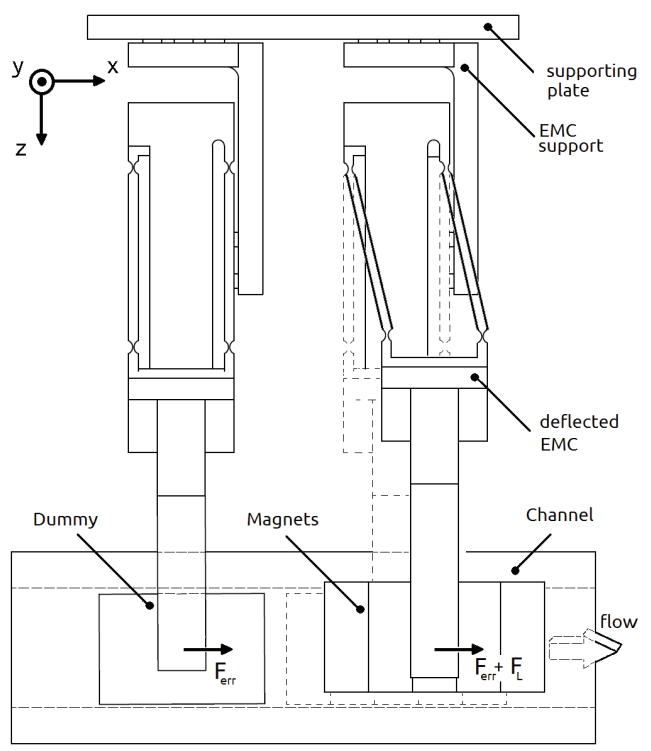

Fig. 4. Schematics of measurement principle. Dashed line in the right indicates initial state of balance unaffected by Lorentz force, later when metering the flow a deflection results (exaggerated representation). In working mode balance is always in 0 position, instead compensation current inside of EMC balance which drives the measurements is the indication of deflection.

The EMC balances are equipped with factory electronic that preforms compensation procedure with well defend cycle of analog PID controller and under various filtering modes available optionally. Measurements were taken from electronics of both EMC balances simultaneously and stored in PC through USB communication port with several $\mu s$ delay. Although electronics provides around 80 to 100 sampling rate we limit ourselves with sufficient enough measurement frequency of 24 to $26 \mathrm{~Hz}$, since a) the settling time of EMC balance itself is somewhat about $1.4 \mathrm{~s}$ and $\mathrm{b}$ ) measurement of Lorentz forces yet is in order of up to several $\mathrm{Hz}$ frequencies. Below presented (4a-c) calculation principle reveals basic principle underlying in our measurements

$$
\begin{aligned}
& F_{M 1}=F_{L}+F_{T}+F_{G V}=F_{L}+F_{e r r} \\
& F_{M 2}=F_{T}+F_{G V}=F_{e r r} \\
& F_{M 1}-F_{M 2}=F_{L}
\end{aligned}
$$

The model is idealized since we consider case of alignment along $X$ axis, neglecting all other influences and that system response only to $X$ component of each force. Here, $F_{M 2}{ }^{-}$is indication of force signal detected by first balance that consist $F_{T}$ - tilt effect and $F_{G V}$ ground vibration effects, last two existing in the signal of second balance given as $F_{M 1}$ which detects influences of Lorentz force $F_{L}$ as well. The system in the preliminary development state has shown already capability to resolve distinguished force measurements around 100 $\mathrm{nN}$ and below with approximate standard deviation of 20 to $30 \mathrm{nN}$ dependent from filtering and operating mode (sees Fig. 5). Below is typical response of system against calibration forces given by linear voice coil motor [11]. The magnitude, direction and frequency of the force are chosen as identic as the presumable character of the Lorentz force predicted to be measured.
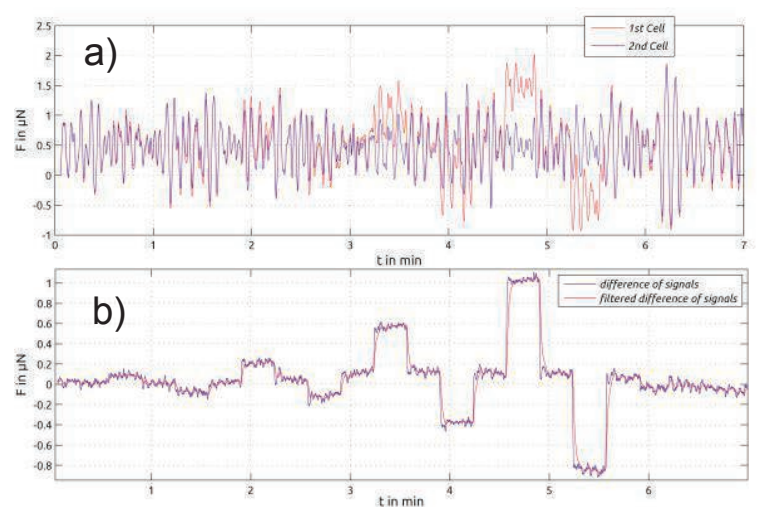

Fig. 5. a) Raw response signal of both $F_{M 1}$ and $F_{M 2}$ EMC balances against minimal calibration forces up to $1 u N, b)$ difference of signals.

Notice that single EMC balance has clearly observable noise that defines measurements resolution of several hundreds of $\mathrm{nN}$. This noise is purely mechanical $F_{G V}$ which exist in both signals. Shown in short term measurements 7 minutes (see Fig. 5) this systematic noise is possible to eliminate. Unlike thermal influences which are leading system to tilt (discussed earlier) it is experimentally observed that for long term measurement the vibrational noise can be entirely eliminated. Since the tilt $F_{T}$ has multiple axes appearance in the system, it was observed in our measurements only after short term up to 20 - 30 minute period the divergence of measurements from common $X$ axis. Hence, for short term measurement period we have achieved relatively stable measurement signal for different 3 orders of magnitude forces $10^{-8}$ to $10^{-5}$ with the lowest achieved standard deviation $2 \times 10^{-8} \mathrm{~N}$ (notice 
that ours are dynamic measurements while factory standard is given as $\approx 1 \times 10^{-8} \mathrm{~N}$ readability for static measurements). This advance in force measurements, compared with previous work in [7] where signal was not stable and resolution of measurements were limited to $1 \times 10^{-6} \mathrm{~N}$, let as to make further investigations in the actual application of interest LFV measurements. Procedure and results of metering velocity of electrolyte flow in rectangular channel is presented next

\section{Experimental procedure and measurement results}

Channel has around $150 \mathrm{~cm}$ length throughout which is circulating $\approx 300 \mathrm{~L}$ electrolyte solution (see Fig. 6).

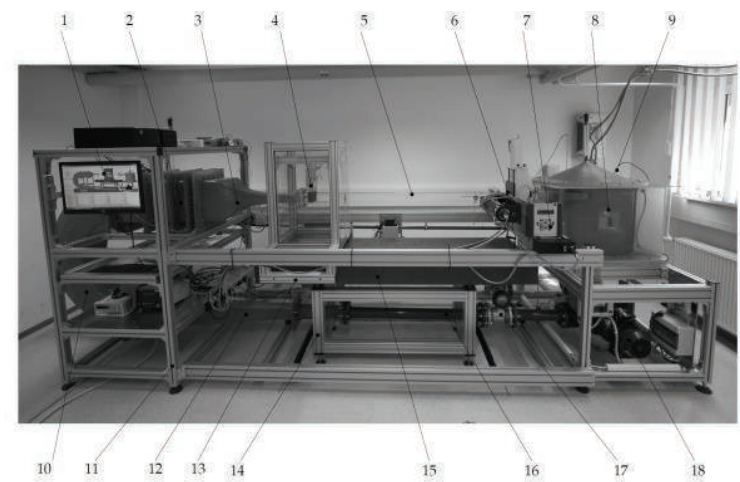

Fig. 6. 1-PC control, 2-Nozzel of the channel, 4EMC system, 5-Channel, 6-LDA, 7-UDV, 8Electrolyte vessel, 9-Conductivitymeter, 13Aluminium housing, 14-Aluminium table, 15-Granits block, 17-MID reference velocitymeter, 18-Pump. Adapted from [12].

For experimental convenience tap water has been chosen as initial electrolyte. On base of existing value of conductivity further certain amount of a conventional table salt have been added to set required, the next value of conductivity. Here we will present collection of Lorentz force measurements for conductivity in different ranges and orders of magnitudes, particularly $\sigma=[0.03 ; 0.3 ; 1 ; 3 ; 10] \mathrm{S} / \mathrm{m}$. Accuracy of online conductivity measurements is given as standard deviation of $\approx \pm 0.002 \mathrm{~S} / \mathrm{m}$.

For each set of conductivity, we have applied stepwise function of velocity with $\approx 0.011 \mathrm{~Hz}$ and collected dates of measurements after settling time is achieved. Since the settling time of entire process is not yet defined due to its complex magnetohydrodynamic character we make assumption on that by observing both force and reference velocity signal until they become stable. Typically maximum time to observe stable signal is $\approx 10$ to 15 s with $\approx 10 \%$ overshoot (see Fig. 7).

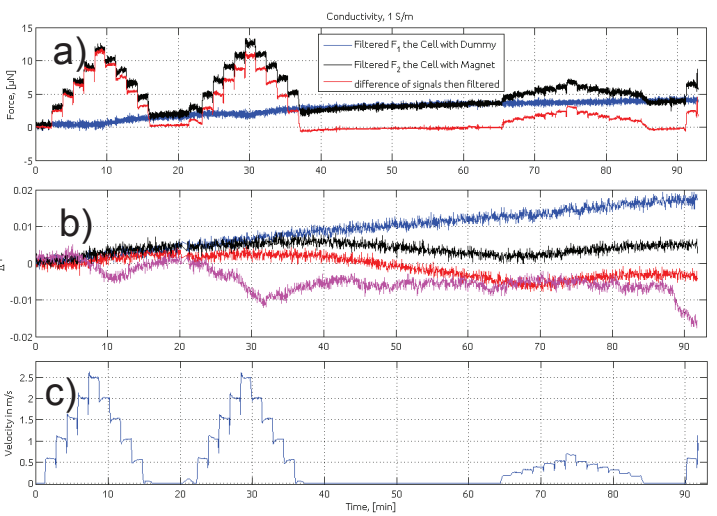

Fig. 7. a) Filtered Force signals and their difference, b) temperature change of ambient environment inside and outside of the housing, c) velocity signal given by reference MID velocitymeter.

The experimental setup yet to be tested for rapid regimes of operation up to $0.5 \mathrm{~Hz}$. However, results of measurements as it was discussed above are depicted in Fig. 8 in terms of measured Lorentz force against reference velocitymeter.

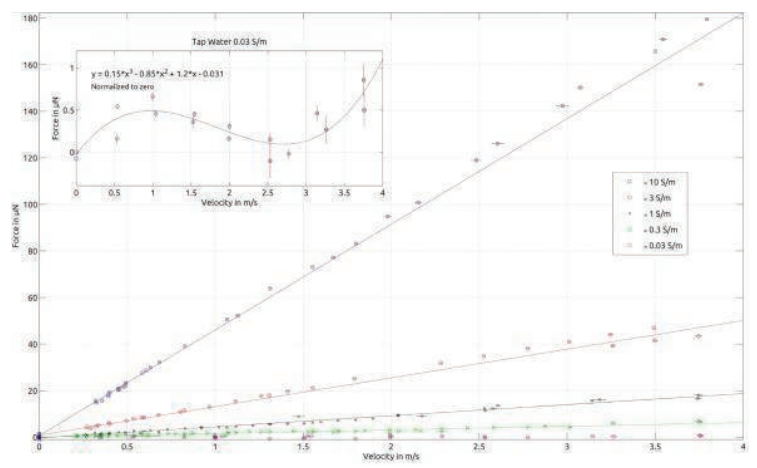

Fig. 8. Lorentz force response against reference MID velocitymeter values and linear fits (solid line), inset: magnification of tap water measurement results fitted by polynomial and normalized to zero.

There are certain nonlinear effects in measurements of tap water which are currently under investigation.

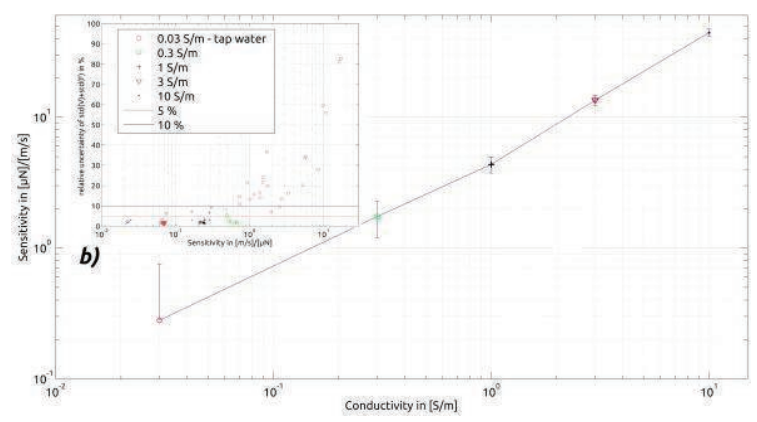

Fig. 9. The sensitivity of setup as relation between ratio of the Lorentz force to the velocity against electrical conductivity, fitted towards the means. Inset: relative uncertainty of measurements in \% against the sensitivity in $\mu \mathrm{N} / \mathrm{m} / \mathrm{s}$.

However, based on presented results we can draw a fractional dependence of measured 
Lorentz force to velocity rate against each value of conductivity that measurement was taken (see Fig. 9). Shown dependence can be interpreted as a sensitivity diagram of LFV flow meter, particularly choosing a conductivity range from graph one can define range of the forces that can be expected to measure, and accordingly adjust range of force measurement devise. Inset Fig.9 shows accuracy of our system based on uncertainty consideration of each measurement device. Also, the measurements above range of conductivities $0.3 \mathrm{~S} / \mathrm{m}$ can be conducted within $5 \%$ combined uncertainty for velocities as small as 0.2 - 2 $\mathrm{m} / \mathrm{s}$. For tap water $(0.03 \mathrm{~S} / \mathrm{m})$ measurements samples are spread somewhat in between 10$50 \%$, which is attributed mostly to rotational and other two $y$ and $z$ components of force vector originating from tilting error. As it was emphasized in (2) the calibration factor can be revealed, combining whole measurements together from sensitivity curve in form of $F_{L} / \sigma \cdot v_{0}$ and taking in account upper and lower limits of uncertainties for each conductivity in range from 0.3 to $10 \mathrm{~S} / \mathrm{m}$ we get value of $c \cdot B^{2}$ as $4.75 \pm 0.61$ that $12 \%$ of standard deviation from the value (see Fig. 10).

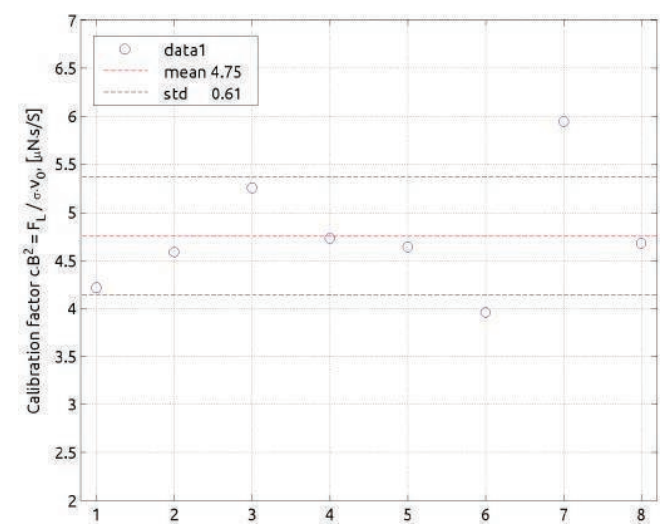

Fig. 10. Calibration factor given in $\mu N s / S$. Note that magnetic field is given together with calibration factor because its value is assumed to be constant. In all measurements have been used the same Halbach array.

\section{Conclusion}

It is evident from results and from the accuracy of measurements that setup has still several aspects to investigate critically, currently in progress to be resolved. a) The long term stability problem, this can be done by considering effects of influentially high thermal fluctuations on both supporting constructions and EMC balance. Also it is desirable to choose a special type of supporting construction and materials with low thermal expansion coefficient. b) A set of measurements are desirable to explore the limits of frequency response of LFV measurement. c) More measurements in the low conductivity and low velocities ranges are necessary. As a conclusion we present in the paper a differential force measurement system for horizontal forces in range of below $10^{-7}$ to $10^{-5} \mathrm{~N}$ with $2 \times 10^{-8} \mathrm{~N}$ resolution. With use of the setup a flow measurement were conducted in rectangular channel by $50 \times 50 \mathrm{~mm}$ cross section for conductivities of $[0.03 ; 0.3 ; 1 ; 3 ; 10] \mathrm{S} / \mathrm{m}$ and in range of 0.2 to $4 \mathrm{~m} / \mathrm{s}$ velocities. A sensitivity curve was obtained, also combined uncertainty and calibration factor of measurement setup have been provided.

\section{References}

[1] A. Thess, E. Votyakov, and Y. Kolesnikov, Phys. Rev Lett. 96, 164501 (2006); doi: 10.1103/PhysRevLett.96.164501

[2] J. A. Shercliff, The Theory of Electromagnetic Flow Measurement. Cambridge University Press, 1962

[3] S. Vasilyan and T. Froehlich, "Direct Lorentz force compensation flowmeter for electrolytes," Applied Physics Letters, vol. 105, no. 22, 2014. DOI:10.1063/1.4903235

[4] B. Halbedel, C. Resagk, A. Wegfrass, C. Diethold, M. Werner, F. Hilbrunner, and A. Thess, "A novel contactless flow rate measurement device for weakly conducting fuids based on lorentz force velocimetry," Flow, Turbulence and Combustion, vol. 92, no. 1-2, pp. 361-369, 2013. DOI:10.1007/s10494-013-9505-5

[5] A. Wegfraß C. Diethold, M. Werner, T. Froehlich, B. Halbedel, F. Hilbrunner, C. Resagk, and A. Thess, Applied Physics Letters 100, 194103 (2012); doi: 10.1063/1.4714899

[6] A. Wegfraß., C. Diethold, M. Werner, C. Resagk, T. Fröhlich, B. Halbedel, A. Thess: Flow rate measurement of weakly conducting fluids using Lorentz force velocimetry. Meas. Sci. Technol. 23, 105307 (2012) doi:10.1088/09570233/23/10/105307

[7] C. Diethold and F. Hilbrunner, Meas. Sci. Technol. 23074017 (2012) doi:10.1088/09570233/23/7/074017

[8] Sartorius Weighing Technology $\mathrm{GmbH}$, Model WZA16-LC, 2013

[9] Leica Geosystems, model: Nivel 210

[10] COMSOL Multiphysics $®$. 2011. Version 4.2. COMSOL, Inc., Burlington, MA, USA.

[11] Linear Voice Coil Motor HVCM-016-010-003-01 Moticont

[12] A. Wegfraß, Doctoral Dissertation, 2013, „Experimentelle Untersuchungen zur Anwendbarkeit der Lorentzkraft-Anemometrie auf schwach leitfähige Fluide" 